\title{
Hands-on experimental and computer laboratory in optics: the Young double slit experiment
}

H. Ghalila, A. Ammar, Y. Majdi, S. Lahmar, Z. Dhaouadi, et al.

H. Ghalila, A. Ammar, Y. Majdi, S. Lahmar, Z. Dhaouadi, M. Zghal, Zohra Ben Lakhdara, V. Lakshminarayanan, "Hands-on experimental and computer laboratory in optics: the Young double slit experiment," Proc. SPIE 10741, Optics Education and Outreach V, 107410F (14 September 2018); doi: $10.1117 / 12.2322164$

EDIE Event: SPIE Optical Engineering + Applications, 2018, San Diego, California, United States 


\title{
Hands-on experimental and computer laboratory in optics: The Young Double Slit Experiment
}

\author{
H. Ghalila, ${ }^{\mathrm{a}, \mathrm{b}}$, A. Ammar ${ }^{\mathrm{a}, \mathrm{b}}$, Y. Majdi ${ }^{\mathrm{a}, \mathrm{b}}$, S. Lahmar, ${ }^{\mathrm{a}, \mathrm{b}}$, Z Dhaoudi $^{\mathrm{a}, \mathrm{b}}$, M. Zghal ${ }^{\mathrm{b}, \mathrm{c}}$, \\ Zohra Ben Lakhdar ${ }^{\mathrm{a}, \mathrm{b}}$, V. Lakshminarayanan ${ }^{* \mathrm{~d}}$. \\ ${ }^{a}$ Laboratoire de Spectroscopie Atomique, Moléculaire et Applications, Faculté des Sciences \\ de Tunis - Université de Tunis El Manar, Tunis, Tunisia, 'Société Tunisienne d'Optique, \\ Tunis, Tunisia, ${ }^{\mathrm{C}}$ University of Carthage, Engineering School of Communication of Tunis \\ (Sup'Com), GreS'Com Laboratory, Ghazala Technopark, 2083 Ariana, Tunisia, \\ ${ }^{\mathrm{d}}$ Theoretical and Experimental Epistemology Lab, School of Optometry and Vision \\ Science, University of Waterloo, Waterloo, ON N2L 3G1, Canada
}

\begin{abstract}
Teaching optics to small groups of students allows them to share ideas and leads to discussions, which will enable them to understand concepts better. This is a form of peer teaching/evaluation. This group dynamic favors creativity and inhibits obstacles to learning and understanding due to shyness, and other psychological factors. In addition, this paradigm allows the learner to be an active participant in the learning process rather than a passive recipient of knowledge as in the traditional lecture based teaching methodology. The project proposed here is based on both experimental and numerical approaches. Groups of students will be using simple and inexpensive equipment in a hands-on way. Additionally using numerical tools with open source environments such as the Python programming language allows one to perform numerical experiments. These two approaches are perfectly complementary; indeed the experiments favor observations and measurements and on the other hand numerical modeling favors abstraction and familiarization of mathematical formalisms of the optical phenomena. We propose a pedagogical methodology "Active Learning in Simulating Optics" (ALSO), where the active learning method is used not only for hands on experimentation while numerical modeling facilitates development of computer codes wherein students can design their own experiments. Mixing these two approaches, experimentation and simulation, is also very well adapted in working within projects for the elaboration of a new tools for teaching. This ALSO methodology will be presented along with results from workshops utilizing this technique.
\end{abstract}

Keywords: Active learning, Optics simulations, Peer teaching, Numerical experiments, Open source Python, physical optics.

\section{INTRODUCTION}

Learning fundamental concepts in the context of a more extensive project including various aspects of pedagogy, experimentation, numerical modeling and also legislative (standards) and marketing aspects, is an attractive framework for many high school students and undergraduates. Optics is well suited for this approach due to its ubiquity in our daily life, its simplicity for experimental observations and also the relatively easy form of the analytical expressions, which are used to model direct observations. The key point is the enormous sense of accomplishment students feel when starting from a direct observation and end up example a usable applet on smartphone. This type of pedagogical process is extensively participatory and interactive for both students and teachers. Of course the idea is not to copy one of the numerous online codes already existing in the web and then implement them in the project (see for example references 17-20 in Reference ${ }^{[1]}$ and related discussion). If this was the case, the students become simple passive consumers. We should also be extremely mindful of the economic costs associated with such activities. For this reason projects should be based on inexpensive

Optics Education and Outreach V, edited by G. Groot Gregory, Proc. of SPIE Vol. 10741, 107410F

(C) 2018 SPIE · CCC code: 0277-786X/18/\$18 - doi: 10.1117/12.2322164 
equipment for experimentation and open source software for numerical modeling. This is a very important requirement for teaching in developing countries. In terms of software this is easily achievable with programming environments such as Python which allows designing powerful software that are compatible with many OS (Linux, Windows, iOS).

We present here a continuation of our previous studies on the pedagogical methodology "Active Learning in Simulating Optics" (ALSO) and the role of simulations in optics educations using the Python language ${ }^{[2-5]}$. We describe here a new version of the program developed to accompany the direct observation of interference-diffraction phenomena obtained by hand-made double slits and an inexpensive laser source. The resulting tool is powerful software that can deal with very small sizes (fraction of $\mathrm{mm}$ ) for the slits width or the distance between them.

\section{LABORATORY MANIPULATIONS}

The first step before starting the project is to choose the appropriate topics that will lead to the ultimate goal in terms of acquiring fundamental concepts, training and building the software. Many examples are possible from the multitude of setups generally studied throughout the introductory courses on optics. We choose here the Young double slit experiment for the study of the interference-diffraction phenomenon because of its simplicity in in terms of experimental implementation and also the simplicity of the analytical solution. As a side note, the double slit experiment appears twice in a survey of the top 10 beautiful experiments of all time (at \#1 for the double slit experiment done with electrons and at $\# 5$ for the double slit light interference experiment). This survey was conducted amongst reader of the magazine Physics World published by the Institute of Physics, $\mathrm{UK}^{[6]}$.

\subsection{Learning through experimentation}

Great efforts were made to reduce the equipment necessary for producing interference-diffraction pattern. This was achieved by constructing a kit made up of (1) a small square of glass painted in one side on which two parallel thin slits were made using a blade/cutter and (2) an inexpensive laser pointer costing about a dollar (Figure.2-left). A blank Wall is used as the screen. The most expensive tool is probably the camera, but it is likely that at least one is available in the class (e.g., good resolution smartphone camera). The two slits are easy to prepare but the result is not guaranteed so students are encouraged to prepare different configurations, which are used for comparison. The students are broken up into a number of groups of say 4 students each. Normally students in introductory classes do not have a precise idea of the expected results so during the class the different groups are called to discuss their ideas. In this context the teacher also has to change the classical and usually passive way of teaching to one favoring Predictions, Observations, Discussions and Syntheses (PODS).

This method of teaching (PODS) has been investigated for many years under the framework of the UNESCO project "Active Learning in Optics and Photonics" (ALOP) ${ }^{[7]}$ with the support of ICTP (Abdus Salam International Centre for Theoretical Physics, Trieste, Italy) and generously supported over the years by SPIE (SPIE - The International Society for Optics and Photonics). Many workshops have been conducted throughout the word. The main point in this framework is to focus on learning exclusively based on experimental observations favoring PODS and avoiding as far as possible mathematics and abstract formalisms. The intent is conceptual understanding of the physics behind the observations.

\subsection{Data acquisition}

Special care should be paid when acquiring data because the observed pattern could change drastically from one measurement to another. For this reason, students are encouraged to capture numerous pictures of the light observed on the wall. Figure.1 illustrates some of the pattern usually obtained by our simple equipment. 

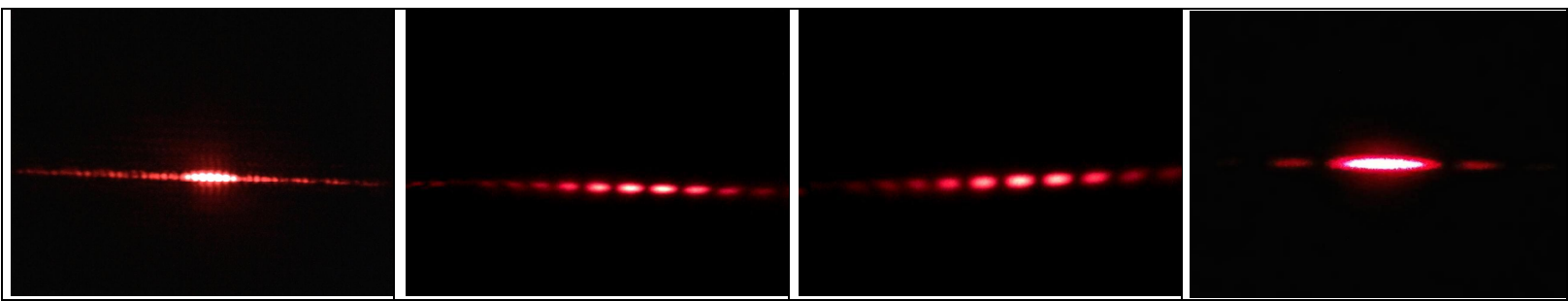

Figure.1 : Some of the pictures captured with different configuration of slits. The second and the third one can be convenient for numerical prediction

The first three pictures illustrate the diffraction-interference patterns obtained by the Young double slits. The last figure corresponds to diffraction by a single slit. This last result occurred when the laser source lit only one of the two slits. So students should pay attention to properly illuminate both of the slits. The first picture shows clearly the diffraction pattern with the main central peak accompanied by the secondary ones, but in this case the photographer was too far away and the interference spots are not clearly resolved. The second and third pictures correspond to the same configuration; the only difference is a small tilt of the camera during image acquisition. These two pictures are considered good because interference patterns are clearly resolved and we can also see the effects of diffraction modulating the brightness of the spots. The small tilt between them also reveals the difficulty of perfectly capturing the desired image and makes us aware of the precautions to take when taking photographs. The main disadvantage of these two pictures is that the secondary peaks of diffraction are not included. During class, teachers should alert students about these points, emphasizing that the best photograph will require less manipulation and lead to the best estimation for the slit width and the distance between them.

During this first stage of the project, by changing the slit width and separations as well as the slit-wall distance, the students are encouraged to test a great number of different configurations. For each result/picture, students should register some values (see Figure.2 right) such as the size $\Delta S$ of the main spot measured on the screen and corresponding to the main peak of diffraction. They also need to measure the size of the small spots $\Delta s$ included in the main spot and corresponding to the interference. They should count the number of small spots included in the main one and record it also. The distance from the screen to the plan of the slits $D$ will also be needed for later calculations.
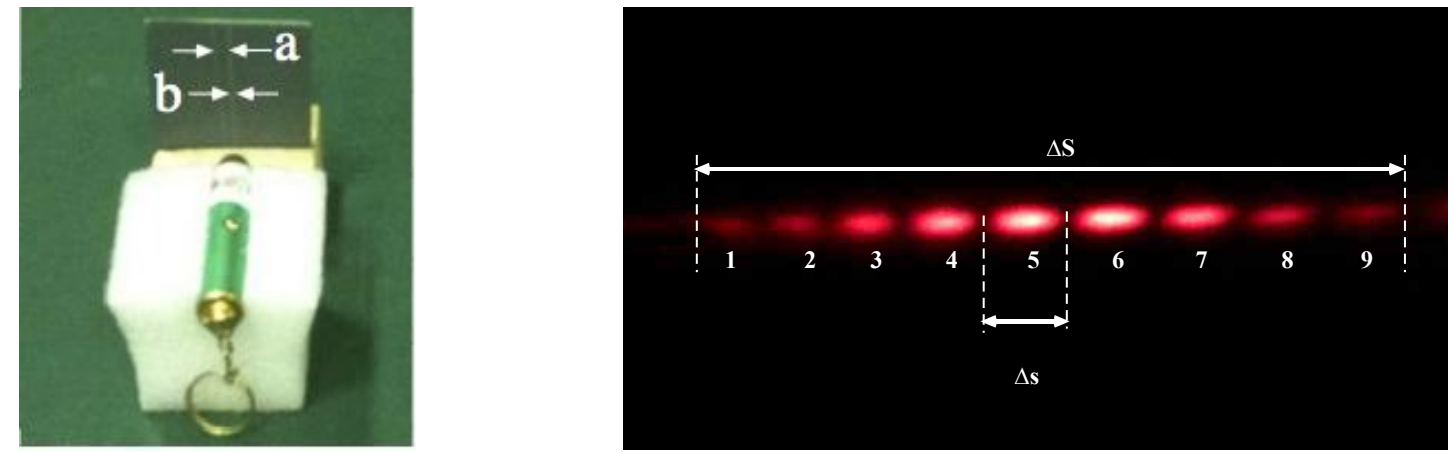

Figure.2 : In the left the experimental setup with the hand-made slits and the laser pointer. In the right the measures of the size of the main central peak of diffraction $\Delta S$, the size of the small spot du to the interferences $\Delta s$ and enumeration of the number of interference peak inside the main peak of diffraction

The distance $D$ acts as an input parameter and except for this parameter, which can be easily varied and measurable, the slit width and the distance between them are made imprecise or even unknown. All these parameters will be used when comparing experiments and numerical modeling and as goal of the experiment is to determine precisely these last two parameters $(\Delta S$ and $\Delta s)$. 


\section{NUMERICAL MODELING}

We are not going to explain here the basics of coding and programming. This is elaborated upon in a recent book ${ }^{[4]}$. Here we will focus on the main goal that we set to ourselves and which is the determination of the slit width and the distance between them.

\subsection{Analytical expression}

The Analytic solution describing the intensity of light obtained with the Young double slit experiment is simply expressed as (see any introductory optics text book):

$$
\mathrm{I}(\mathrm{x})=\operatorname{sinc}^{2}(\mathrm{Bx})[1+\cos (2 \mathrm{Ax})] \quad \text { where }: \mathrm{A}=\pi \mathrm{a} / \lambda \mathrm{D} \text { and } \mathrm{B}=\pi \mathrm{b} / \lambda \mathrm{D} \text {, }
$$

$b$ stands for the width of the slits, $a$ represents the distance between slits, $D$ is the distance of the screen to the plan of the slits and $\lambda$ is the wavelength of the monochromatic incident light. The first term [ $\left.\operatorname{sinc}^{2}(\mathrm{Bx})\right]$ describes the diffraction phenomenon due to a single slit and the second term is associated with the linear superposition between the light waves originating at the two slits. Figure. 3 shows as an example the Intensity obtained with arbitrary values for $b, a, \lambda, D$.

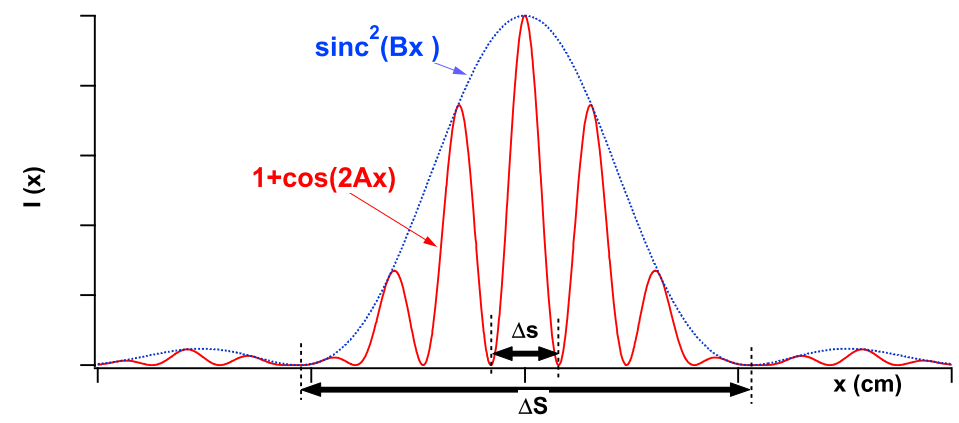

Figure.3 : Simulation of the light intensity produced by Young double slits for arbitrary values $(b=150 \mu \mathrm{m}, \mathrm{a}=0.5 \mathrm{~mm}$, $\lambda=635 \mathrm{~nm}$ and $\mathrm{D}=5 \mathrm{~m})$.

As shown in this figure, the diffraction term is simulated by the blue curve and its main central peak is defined by its width $\Delta S$ delimited by the two surrounding minima. The red curve depicts the interference phenomenon and the size of the small peaks is also defined by their width $\Delta s$. The values of $\Delta S$ and $\Delta s$ are determined theoretically from $b$ and $a$ :

$\Delta \mathrm{S}=2 \lambda \mathrm{D} / \mathrm{b} \quad$ and $\quad \Delta \mathrm{s}=\lambda \mathrm{D} / \mathrm{a}$

Going back to Figure.2 and knowing the measured values of $\Delta S$ and $\Delta s$ and as well their theoretical expression we can determine the values of $b$ and $a$ by fitting the numerical simulation of $\mathrm{I}(\mathrm{x})$ to the experimental one obtained from the picture. This last operation can be done using standard image processing tools available in python environment such as scikit-image (http://scikit-image.org/) or scipy.ndimage (https://docs.scipy.org/doc/scipy-0.14.0/reference/ndimage.html).

\subsection{Curve fitting experimental results}

The main point here is to find a convenient way to reach our goal, namely the determination of $b$ and $a$ knowing $\lambda$ and $\mathrm{D}$, in an easy and convenient manner. For this purpose python language is very well suited and offers a wide range of methods to create and interface programs and to manipulate images and combine them with curves and annotations. We built for this a program composed of two parts, the main one links and displays experimental and theoretical curves and the second one (sub-program) is dedicated to image processing. For details of the programming se $\mathrm{e}^{[4]}$. Figure.4 shows the interfaces of both programs; the interface of the main program (left) from which we can call via the button Image Tool the sub-program (right). 


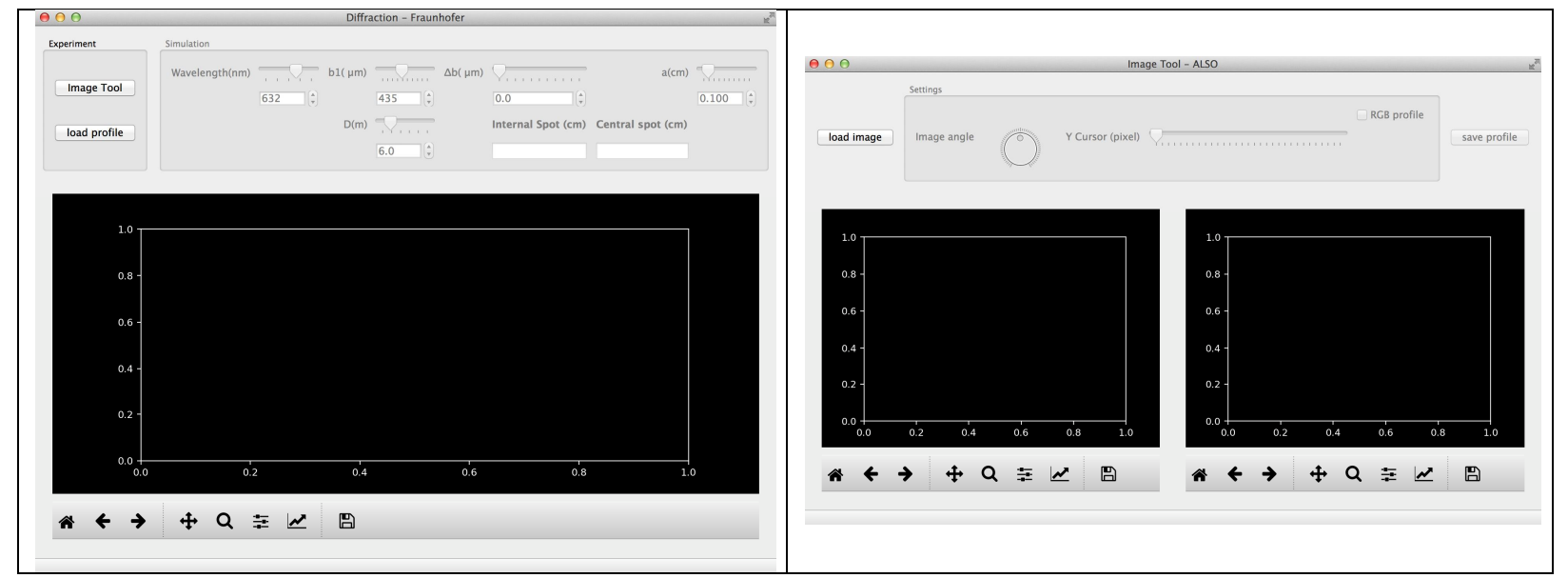

Figure.4 : On the left, the interface of the main program used to superimpose experimental intensity profile and the simulated one and on the right the secondary program for the creation of the intensity profile.

By clicking on the button Image Tool, the users are invited to open the desired picture via the second interface Load Image to generate the desired 'Intensity profile'. For example we can open the picture displayed in Figure.2. After that, as illustrated in Figure.5 below, with the help of the button Image angle and the slider $Y$ cursor, the users can bring the yellow dashed line to the position of the diffraction-interference pattern with the right orientation. This dashed line keeps the luminosity profile of the image and displays it directly in the second screen.

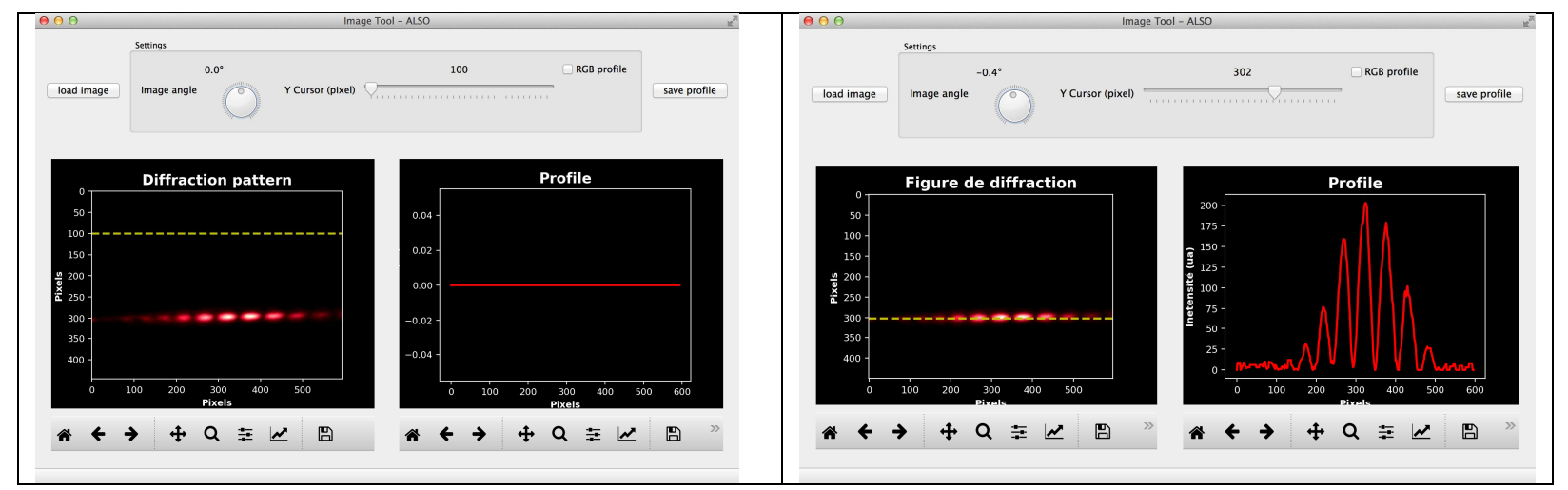

Figure.5 : Interface of the image processing program. In left the startup configuration after loading image and in right after keeping the desired profile

After saving the desired profile, it is possible to load it in the main interface as shown in Figure.6 (left) and with the help of the various widgets (button and sliders) implemented in the program it is possible to vary interactively the physical parameters such as the wavelength, the slit width the distance between them and the distance $(b, a, \lambda, D)$ in a way to adjust as much as possible the experimental profile (red curve) with the theoretical curve (yellow curve). 


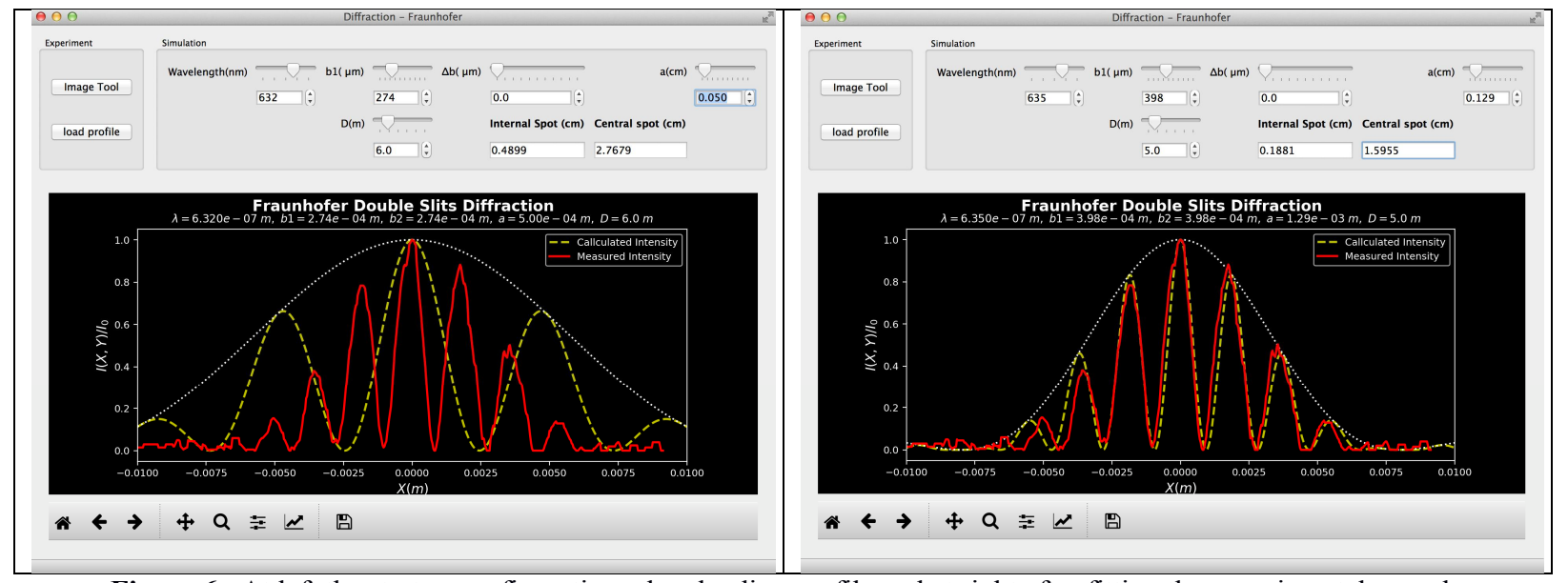

Figure.6 : At left the startup configuration when loading profile and at right after fitting the experimental record.

Simultaneously with the manipulation of the various widgets used for adjusting the theoretical curve with the experimental one, the program displays on the same panel the value of the width of the main spot or central spot $\Delta S$ and the value of the small spots or internal spots. For example, we can read in this case $\Delta S=1.595 \mathrm{~cm}$ and $\Delta s=0.188 \mathrm{~cm}$ and this for a diffraction-interference pattern obtained for monochromatic light of $\lambda=635 \mathrm{~nm}$ incident on two slits of width $b=398 \mu \mathrm{m}$ separated by $a=0.129 \mathrm{~cm}$ and observed on the wall of $D=5 \mathrm{~m}$ from the plane of the slits.

So we can use several criteria to decide the correctness of the fitting,

- the sinc function (white curve in Figure.6) should cover the tops of the interference fringes as best as possible

- the number of the fringes enclosed in the mean peak should be same for experiment and simulation

- and most importantly, the values of both widths $\Delta S$ and $\Delta s$ should be identical to those directly measured in the wall

All these considerations should give us a good estimation of the two parameters $b$ and $a$ hand-made by students. In the case the width of the two slits differs, which is likely the case, it is also possible to vary one of the two slits ( $\Delta b$ in Figure.6) keeping the other constant. This is also a good opportunity to discuss experimental error analysis.

\section{CONCLUSION}

In this paper we have shown how easily conducted experiments and computational methods can be used to teach important ideas in optics. Starting from a simple observation with the help of very simple and hand-made equipment and by learning computer programming with an open source environment such as python we can give a deeper understanding of the fundamental concepts in optics and at the same time develop new tools for use by teachers and students. For instance, the program developed in this work can be easily implemented in smartphones as an applet. Nowadays, even in developing countries, where smartphones are widely used, it becomes possible to reorient the classical classroom environment to one that is project-oriented. During these classes, students learn fundamental concepts by combining experiment and theory via numerical computing and also realize at the same time the importance of accuracy in taking measurements. Also, they can investigate a wide range of configurations that are not always possible because of cost considerations. 


\section{REFERENCES}

[1] Lakshminarayanan V., H.Ghalila, A.Ammar, Varadharajan, L.S., Role of simulations in optics education, Proc SPIE, Vol. 9946, doi : 10.111/12.2236159 (2016).

[2] Ammar, A., Burman, R., Ghalila, H., Ben Lakhdar, Z., Varadharajan, L.S., Lahmar, S., and Lakshminarayanan. V., "Optics simulations with Python: Diffraction”, Proc. SPIE Vol. 9793, (2015), doi: $10.1117 / 12.2223072$.

[3] Lakshminarayanan, V., and Burman, R.,"Optics Tutorials with Python", Tech. Report, Univ. of Waterloo, 2015, doi: 10.13140/RG.2.1.2940.2325, (2015).

[4] Lakshminarayanan, V., Ghallila, H., Ammar, A., Varadharajan, L.S., Understanding Optics With Python, CRC Press/Taylor and Francis, Boca Raton, FL., (2018).

[5] Ghalila, H., Ammar, A., Varadharajan, S., Majdi, Y., Zghal, M., Lahmar, S., Lakshminarayanan, V., “Optics Simulations: A Python Workshop": Proc. SPIE Vol.10452, doi: 10.1117/12.2268377 (2017).

[6] R.P.Crease, The most beautiful experiment, Physics World, 15(9): 19-21, (2002).

[7] Ghalila, H., Ben Lakhdar, Z., Lahmar, S., Dhouaidi, Z., Majdi, Y., "Active Learning in Optics and Photonics: Fraunhofer diffraction, Proc. SPIE, Vol. 9289, doi: 10.1117/12.2070776 (2014) 